\title{
Values of the bispectral index do not parallel the hemodynamic response to the rapid increase in isoflurane concentration
}

[Les valeurs de l'index bispectral ne correspondent pas à la réponse hémodynamique

à la suite de l'augmentation rapide de la concentration d'isoflurane]

Masayasu Nakayama MD,${ }^{*}$ Michiko Hayashi MD, * Hiromichi Ichinose MD, ${ }^{*}$ Shuji Yamamoto MD,

Noriaki Kanaya MD, $†$ Akiyoshi Namiki MD PhD $†$

Purpose: To investigate the changes in hemodynamic variables and bispectral index (BIS) in response to a rapid increase in isoflurane or sevoflurane concentration.

Method: Thirty adult patients were anesthetized with either isoflurane (isoflurane group) or sevoflurane (sevoflurane group). Two minutes after induction of anesthesia with thiamylal, the inspired concentrations of isoflurane and sevoflurane were rapidly increased from 0.5 minimum alveolar anaesthetic concentration (MAC) to 3 MAC and maintained for five minutes. Heart rate (HR), mean arterial pressure (MAP), and BIS were measured every minute.

Results: An increase in the anesthetic concentration caused increases in HR and MAP in the isoflurane group and a decrease in $M A P$ in the sevoflurane group. Consequently, HR and MAP in the isoflurane group were significantly higher than those in the sevoflurane group. After inhalation of high concentrations, BIS significantly and progressively decreased in both groups.

Conclusion: BIS values decrease after a step increase in volatile agent concentration, whether or not a hyperdynamic action occurs.

Objectif: Examiner les changements des variables hémodynamiques et de l'index bispectral (B/S) en réponse à une augmentation rapide de la concentration d'isoflurane ou de sévoflurane.

Méthode : Trente patients adultes ont été anesthésiés avec de l'isoflurane ou du sévoflurane et ainsi répartis en deux groupes. Deux minutes après l'induction de l'anesthésie avec du thiamylal, les concentrations d'isoflurane et de sévoflurane inspirées ont été rapidement augmentées d'une concentration alvéolaire minimale de 0,5 (CAM) 3 MAC et maintenue pendant cinq minutes. La fréquence cardiaque (FC), la tension artérielle moyenne (TAM) et le BIS ont été mesurés à chaque minute.
Résultats : Une augmentation de la concentration de l'anesthésique a entrainé une augmentation de la FC et de la TAM dans le groupe isoflurane et une baisse de la TAM dans le groupe sévofurane. Par conséquent, la FC et la TAM ont été significativement plus élevées dans le groupe isoflurane que dans le groupe sévoflurane. Après l'inhalation de fortes concentrations, le BIS a diminué de façon progressive et significative dans les deux groupes

Conclusion : Les valeurs du BIS diminuent à la suite d'une augmentation rapide de la concentration de l'anesthésique volatil, qu'une réaction hyperdynamique survienne ou non.

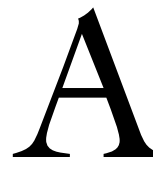

rapid increase in the inspired isoflurane concentration causes transient sympathetic nervous system activation and hyperdynamic circulation. ${ }^{1-6}$ It has been suggested that isoflurane has an irritating effect on the airways that subsequently elicits tachycardia and hypertension. ${ }^{4,7}$ In contrast, sevoflurane does not elicit these hyperdynamic responses, perhaps because it evokes less airway irritation than does isoflurane. ${ }^{8}$

The bispectral index (BIS), obtained from bispectral analysis of the electroencephalogram, has been shown to reflect the hypnotic component of anesthesia. ${ }^{9}, 10$ It has been reported that BIS decreases with increasing concentrations of anesthetics. ${ }^{11-13}$ However, to our knowledge, there have been no reports on the effects of abrupt increases in isoflurane and sevoflurane concentrations on BIS. Since BIS has been shown to be increased by stimuli to the airways, such as endotracheal

From the Division of Anesthesia, Obihiro Kosei Hospital, ${ }^{*}$ and the Sapporo Medical University School of Medicine, $†$ Sapporo, Japan. Address correspondence to: Dr. Masayasu Nakayama, Department of Anesthesiology, Sapporo Medical University School of Medicine, South 1, West 16, Sapporo 060-8543, Japan. Phone: 81-11-611-2111; Fax: 81-11-631-9683; E-mail: miyabi@zc4.so-net.ne.jp Accepted for publication July 3, 2001

Repision accepted August 1,2001 . 
intubation ${ }^{14}$ and mechanical stimulation of the nasal mucosa, ${ }^{15}$ it is possible that the administration of a high concentration of isoflurane will increase BIS. The objective of the present study was to elucidate the changes in hemodynamics and BIS after rapid increases in isoflurane and sevoflurane concentrations.

\section{Methods}

Thirty ASA I patients, 30 to $60 \mathrm{yr}$, scheduled for elective minor surgery with general anesthesia, were enrolled in this study. The study was approved by our local Ethics Committee, and informed consent for participation in the study was obtained from each patient. Patients were randomly assigned, via sealed envelope assignment, to receive either isoflurane (isoflurane group, $n=15$ ) or sevoflurane (sevoflurane group, $n=15$ ). None of the patients had cardiopulmonary or neurological disorders.

No premedication was given before surgery. The electrocardiogram (ECG) (lead II) and hemoglobin oxygen saturation were monitored continuously throughout the procedure. Heart rate (HR) and mean arterial pressure (MAP) were measured by an automatic oscillographic method (Ml008B, Agilent Technologies, Boeblingen, Germany). BIS (version 3.4) was measured continuously on an EEG monitor (Model A1050; Aspect Medical System, Natick, MA, USA) using BisSensor strips (Aspect Medical System). The strips consisted of three pregelled electrodes, two active and one ground. The impedance of each electrode was maintained at less than 2 Kohms. A soft catheter was inserted about $2 \mathrm{~cm}$ into the nostril to monitor end-tidal carbon dioxide and end-tidal concentrations of isoflurane and sevoflurane (Anesthetic Gas Module M1026A; Agilent Technologies). ${ }^{2-4} \mathrm{We}$ assumed minimum alveolar anaesthetic concentration (MAC) values for isoflurane and sevoflurane of $1.15 \%{ }^{16}$ and $1.71 \%,{ }^{17}$ respectively.

After breathing oxygen, general anesthesia was induced with $2 \mathrm{mg} \cdot \mathrm{kg}^{-1}$ thiamylal $i v$ for $15 \mathrm{sec}$, followed by $0.1 \mathrm{mg} \cdot \mathrm{kg}^{-1}$ vecuronium $i v$. Mask inhalation of isoflurane or sevoflurane at 0.5 MAC in $100 \%$ oxygen $\left(6 \mathrm{~L} \cdot \mathrm{min}^{-1}\right)$ via a semiclosed circle system was begun after loss of consciousness (confirmed by loss of eyelash reflex). Ventilation was controlled to maintain an end-tidal carbon dioxide tension of approximately $40 \mathrm{mmHg}$. Two minutes later, the inspired anesthetic concentration was abruptly increased to $3 \mathrm{MAC}$ and maintained for five minutes. The trachea was then intubated and surgery was begun after all measurements were completed. The anesthesiologist ventilating the patient was blinded as to the inhalational anesthetics used for this study.
Heart rate, MAP, inspired and end-tidal anesthetic concentrations, and BIS were recorded before the induction of anesthesia and every minute during the study period. All data are expressed as means \pm SD. Statistical analysis was performed using two-way analysis of variance (between the groups) and repeated-measures analysis of variance (within groups). Post hoc analyses were performed with Fisher's protected least significant difference test. The chi-squared test was used to compare gender differences between the two groups. A $P$ value $<0.05$ was considered statistically significant.

\section{Results}

The two groups were comparable with regard to gender, age, height, and weight. No patient exceeded a normal body mass index by more than $25 \%$. None of the patients had abnormal ECG, cough, or a hemoglobin oxygen saturation of less than 99\%. The changes in the inspired and end-tidal concentrations of isoflurane and sevoflurane are shown in Figure 1. After inhalation of an inspired concentration of 3 MAC, the end-tidal concentration of sevoflurane increased more rapidly than that of isoflurane. The end-tidal MAC values had reached 2.0 and 2.1 in the isoflurane and sevoflurane groups, respectively.

There were no inter-group differences in baseline HR, MAP, and BIS (Figure 2). Anesthetic induction with thiamylal did not change HR and MAP but significantly decreased BIS to around 80 in both groups. The abrupt increase in anesthetic concentration significantly increased HR and MAP in the isoflurane group throughout the five minute period of inhalation except for MAP at five minutes. In contrast, in the sevoflurane group, HR did not change and MAP significantly decreased. Thus, HR and MAP in the sevoflurane group were significantly lower than those in the isoflurane group. Two minutes after the anesthetic concentrations had increased, BIS significantly and progressively decreased in both groups. The decrease in BIS was significantly greater in the sevoflurane group than that in the isoflurane group at four and five minutes after loss of consciousness.

\section{Discussion}

A rapid increase in the inspired isoflurane, but not sevoflurane, concentration from 0.5 MAC to $3 \mathrm{MAC}$ increased HR and MAP. On the other hand, the administration of high concentrations of both anesthetics progressively reduced BIS values.

Several studies have shown that a sudden increase in isoflurane concentration activates adrenosympathetic function, resulting in hyperdynamic circulation (Table). 

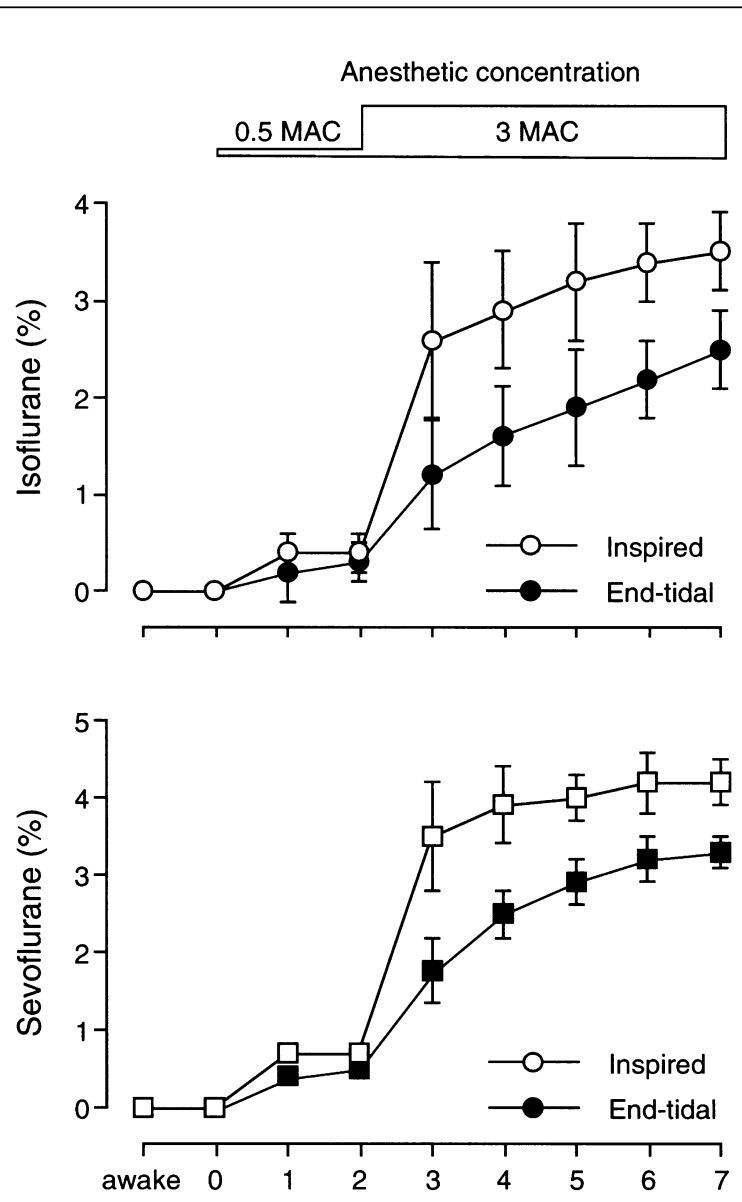

minutes after loss of consciousness

FIGURE 1 Changes in inspired and end-tidal fraction of isoflurane and sevoflurane. Values are mean $+\mathrm{SD}$.

Tanaka et al. ${ }^{4}$ reported that nasal lidocaine considerably inhibited activation of adrenosympathetic function by isoflurane. We have also reported that upper thoracic epidural anesthesia attenuated the circulatory responses to isoflurane more effectively than did lumbar epidural anesthesia. ${ }^{7}$ These results indicate that the pungency of isoflurane stimulates the airway receptors, evoking reflex sympathetic activation. In contrast to isoflurane, a high concentration of sevoflurane did not alter HR but caused MAP to decrease, probably because of its direct effect on vascular smooth muscle. Because sevoflurane is less pungent than isoflurane, ${ }^{18}$ differences in the hemodynamic variables between isoflurane and sevoflurane might have resulted from their different potentials to elicit airway irritation. This finding corresponds to the results of Tanaka et al..$^{8}$ showing that a
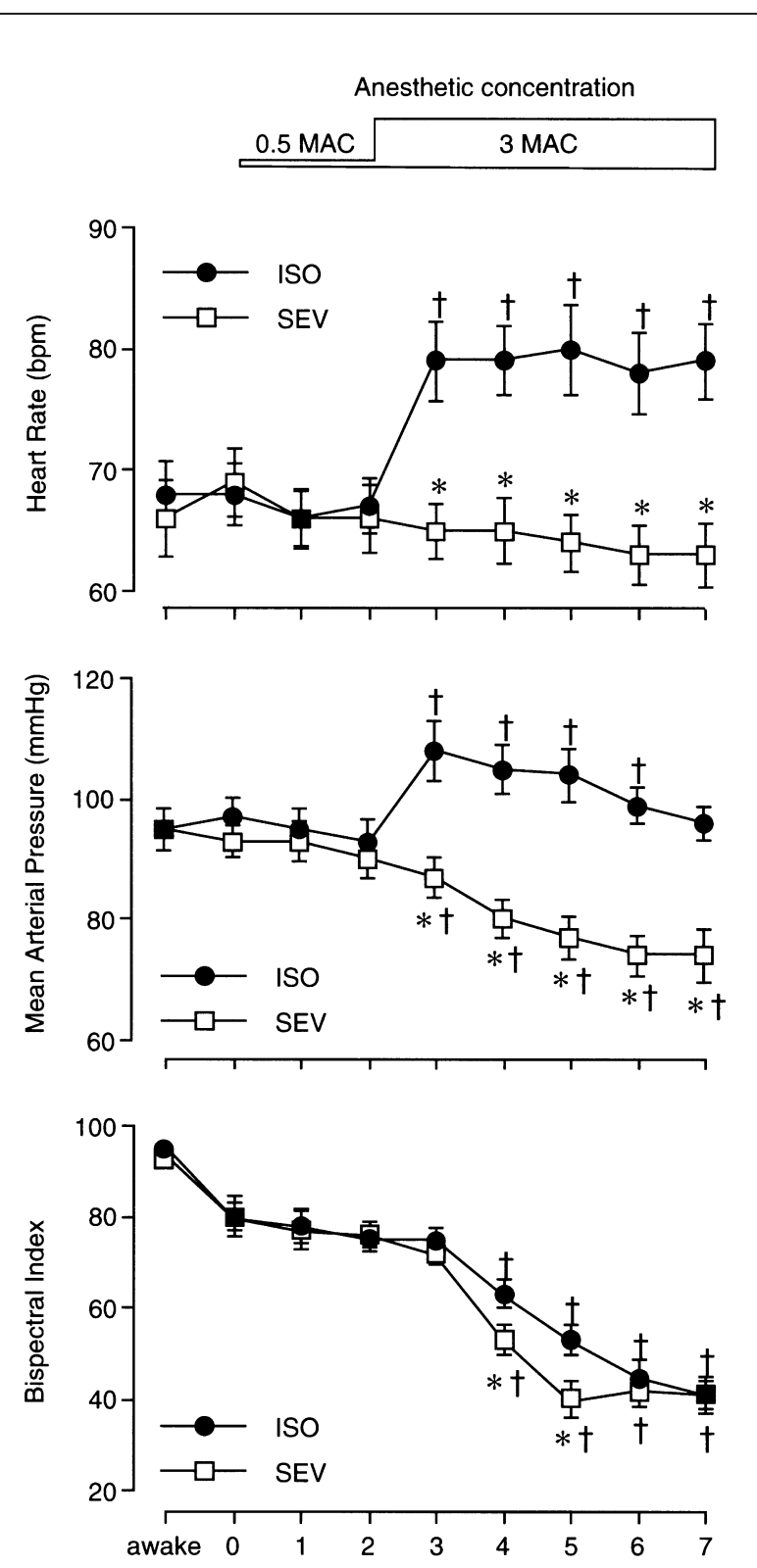

minutes after loss of consciousness

FIGURE 2 Changes in heart rate, mean arterial pressure, and bispectral index after anesthetic induction with thiamylal and increased concentrations of isoflurane and sevoflurane. Values are mean \pm SD. * $P<0.05 v s$ isoflurane group; $\dagger<0.05 v s$ values just before administration of high concentrations of anesthetics.

stepwise increase in isoflurane concentration from 0.9 MAC to 2.7 MAC elicited tachycardia and hypertension but that sevoflurane did not induce hyperdynamic responses. 
TABLE Hemodynamic response to a high concentration of isoflurane in humans

\begin{tabular}{llll}
\hline References & $\begin{array}{l}\text { Maximum } \\
\text { concentration }\end{array}$ & $\begin{array}{l}\text { Rate of } \\
\text { changes in } \\
\text { isoflurane }\end{array}$ & $\begin{array}{l}\text { Hemodynamic } \\
\text { changes }\end{array}$ \\
\hline Randell et al. ${ }^{1}$ & $3 \%$ & Abrupt & $\mathrm{BP} \rightarrow, \mathrm{HR} \uparrow$ \\
Yli-Hankala et al. ${ }^{2}$ & $1.3 \%$ vs. $2.6 \%$ & Abrupt & $\mathrm{BP} \uparrow, \mathrm{HR} \uparrow$ at $2.4 \%$ \\
Ebert and Muzi ${ }^{3}$ & $2.1 \%$ & Steps & $\mathrm{BP} \downarrow, \mathrm{HR} \rightarrow$ \\
Tanaka et al. ${ }^{4}$ & $4 \%$ & Steps & $\mathrm{BP} \uparrow$ (transitory), HR $\uparrow$ \\
Muzi et al. ${ }^{5}$ & $1.8 \%$ & Steps & $\mathrm{HR} \uparrow$ above $1-1.5 \%$ \\
Daniel et al. ${ }^{6}$ & $1.2 \%$ & - & $\mathrm{BP} \downarrow, \mathrm{HR} \rightarrow$ \\
Nakayama et al. ${ }^{7}$ & $5 \%$ & Abrupt & $\mathrm{BP} \uparrow$ (transitory), HR $\uparrow$ \\
\hline
\end{tabular}

Inhalational anesthetics are known to produce dose-dependent effects on BIS. ${ }^{11-13}$ Glass et al. ${ }^{11}$ reported that BIS and the sedation score concomitantly decreased as the end-tidal isoflurane concentration increased from $0.25 \%$ to $1.0 \%$. Katoh et al. ${ }^{12}$ and Demman et al. ${ }^{13}$ reported inverse relationships of BIS to sevoflurane concentration in adult and pediatric patients, respectively. In the present study, we found that even sudden increases in isoflurane and sevoflurane concentrations induced progressive decreases in BIS. The reductions in BIS caused by isoflurane and sevoflurane reached a plateau at around 40 . This finding agrees with the results of Olofsen $e t a l .{ }^{19}$ showing no further decrease in BIS at about 40 with increases in isoflurane and sevoflurane concentrations beyond $0.75 \%$ and $1.5 \%$, respectively.

The end-tidal concentration of sevoflurane increased more rapidly than that of isoflurane because of differences in the blood/gas solubilities. This could explain the difference in changes in not only hemodynamics but also BIS following administration of high concentrations of the two anesthetics. Detsch et al. ${ }^{20}$ reported that an increase in the end-tidal isoflurane concentration from $0.8 \%$ to $1.6 \%$ induced a paradoxical increase in BIS in $40 \%$ of patients. In our study, however, all patients showed progressive decreases in BIS following an increase in isoflurane concentration. Detsch et al. ${ }^{20}$ speculated that the paradoxical increase in BIS is related to continuous pre-burst EEG patterns. Burst suppression occurs at 1.5 MAC of isoflurane, and an isoelectric pattern appears at $2 \mathrm{MAC} .{ }^{21} \mathrm{In}$ the current study, since the end-tidal isoflurane concentration quickly reached 1.5 MAC, the duration of the pre-burst EEG state was probably too short to affect BIS values.

The afferent nerves from irritation receptors in the airways consist of the vagus and the sympathetic nerves to the first four or five thoracic segments. ${ }^{7}$ It has been suggested that a reflex response to a noxious stimulus to the airway is mediated at the subcortical level ${ }^{14,15}$ and thus may be unrelated to the state of consciousness. ${ }^{11}$ Therefore, the BIS value, which reflects cerebral cortical activity, is not influenced by noxious stimuli. However, peripheral noxious stimuli reach the brain through the ascending reticular activating systems of the brain stem. ${ }^{22}$ It has been demonstrated that laryngoscopy and endotracheal intubation increased BIS during infusion of propofol. ${ }^{14}$ However, in the present study, there was no increase in BIS following the sudden increase in isoflurane concentration. This result suggests that the airway-irritating effect of isoflurane is not strong enough to activate the cerebral cortex.

Our use of thiamylal for induction of anesthesia may have influenced the circulatory and BIS changes after anesthetic inhalation. We previously reported that $4 \mathrm{mg} \cdot \mathrm{kg}^{-1}$ thiamylal for induction of anesthesia had less effect on the hyperdynamic responses to a sudden increase in isoflurane concentration than did 2 $\mathrm{mg} \cdot \mathrm{kg}^{-1}$ propofol. $^{23}$ Moreover, Flaishon et al. ${ }^{24}$ reported that recovery of consciousness was achieved approximately five minutes after administration of 4 $\mathrm{mg} \cdot \mathrm{kg}^{-1}$ thiopentone. As we used a smaller dose $(2$ $\mathrm{mg} \cdot \mathrm{kg}^{-1}$ ) of thiamylal than the doses used in these studies, we suspect that thiamylal had only a minimal effect on the current results.

Although previous studies reported that BIS values at loss of consciousness were usually below $65,{ }^{11-13}$ anesthetic induction with thiamylal decreased BIS to around 80 at loss of eyelash reflex in the present study. This finding agrees with the result by Flaishon et al. ${ }^{24}$ who reported that mean BIS at loss of consciousness after rapid $(20 \mathrm{sec})$ administration with propofol and thiopentone were $89 \pm 9$ and $90 \pm 13$, respectively. Since we also administrated thiamylal quickly $(15 \mathrm{sec})$, this may explain why BIS was around 80 at loss of eyelash reflex. Alternatively, there might be a limitation of the applicability of BIS to administration of an induction agent that was not included in the development of the algorithm.

In conclusion, a rapid increase in the inspired concentration of isoflurane, but not that of sevoflurane, was associated with hyperdynamic circulatory responses. However, isoflurane and sevoflurane showed similar progressive and significant reductions in BIS values. These results indicate that BIS values decrease after a step increase in volatile agent concentration, whether or not a hyperdynamic action occurs.

\section{References}

1 Randell T, Seppälä T, Lindgren L. Isoflurane in nitrous oxide and oxygen increases plasma concentrations of 
noradrenaline but attenuates the pressor response to intubation. Acta Anaesthesiol Scand 1991; 35: 600-5.

2 Yli-Hankala A, Randell T, Seppälä T, Lindgren L.

Increases in hemodynamic variables and catecholamine levels after rapid increase in isoflurane concentration. Anesthesiology 1993; 78: 266-71.

3 Ebert TJ, Muzi M. Sympathetic hyperactivity during desflurane anesthesia in healthy volunteers. A comparison with isoflurane. Anesthesiology 1993; 79: 444-53

4 Tanaka S, Tsuchida H, Namba H, Namiki A. Clonidine and lidocaine inhibition of isoflurane induced tachycardia in humans. Anesthesiology 1994; 81: 1341-9.

5 Muzi M, Lopatka CW, Ebert TJ. Desflurane-mediated neurocirculatroy activation in humans. Effects of concentration and rate of change on responses. Anesthesiology 1996; 84: 1035-42.

6 Daniel M, Weiskopf RB, Noorani M, Eger II EI. Fentanyl augments the blockade of the sympathetic response to incision (MAC-BAR) produced by desflurane and isoflurane. Desflurane and isoflurane MAC BAR without and with fentanyl. Anesthesiology 1998 88: 43-9.

7 Nakayama M, Tsuchida H, Kanaya N, Namiki A. Effects of epidural anesthesia on the cardiovascular response to a rapid increase in isoflurane concentration. J Clin Anesth 2000; 12: 14-8.

8 Tanaka S, Tsuchida H, Nakabayashi K, Seki S, Namiki $A$. The effects of sevoflurane, isoflurane, halothane, and enflurane on hemodynamic responses during an inhaled induction of anesthesia via a mask in humans. Anesth Analg 1996; 82: 821-6.

9 Sebel PS, Lang E, Rampil IJ, et al. A multicenter study of bispectral electroencephalogram analysis for monitoring anesthetic effect. Anesth Analg 1997; 84: 891-9.

10 Iselin-Chaves IA, Flaishon R, Sebel PS, et al. The effect of the interaction of propofol and alfentanil on recall, loss of consciousness, and the bispectral index. Anesth Analg 1998; 87: 949-55.

11 Glass PS, Bloom M, Kearse L, Rosow C, Sebel P, Manberg $P$. Bispectral analysis measures sedation and memory effects of propofol, midazolam, isoflurane, and alfentanil in healthy volunteers. Anesthesiology 1997; 86 836-47.

12 Katoh T, Suzuki A, Ikeda K. Electroencephalographic derivatives as a tool for predicting the depth of sedation and anesthesia induced by sevoflurane. Anesthesiology 1998; 88: 642-50.

13 Denman WT, Swanson EL, Rosow D, Ezbicki K, Connors PD, Rosow CE. Pediatric evaluation of the bispectral index (BIS) monitor and correlation of BIS with end-tidal sevoflurane concentration in infants and children. Anesth Analg 2000; 90: 872-7.
14 Mi W-D, Sakai T, Takabashi S, Matsuki A.

Haemodynamic and electroencephalograph responses to intubation during induction with propofol or propofol/fentanyl. Can J Anaesth 1998; 45: 19-22.

15 Mi WD, Sakai T, Singh H, Kudo T, Kudo M, Matsuki $A$. Hypnotic endpoints vs. the bispectral index, 95\% spectral edge frequency and median frequency during propofol infusion with or without fentanyl. Eur J Anaesth 1999; 16, 47-52.

16 Stevens WC, Dolan WM, Gibbons RT, et al. Minimum alveolar concentration (MAC) of isoflurane with and without nitrous oxide in patients of various ages. Anesthesiology 1975; 42: 197-200.

17 Katoh T, Ikeda $\mathrm{K}$. The minimum alveolar concentration (MAC) of sevoflurane in humans. Anesthesiology 1987; 66: 301-3

18 TerRiet MF, DeSouza GJA, Jacobs JS, et al. Which is most pungent: isoflurane, sevoflurane or desflurane? $\mathrm{B}$ J Anaesth 2000; 85: 305-7.

19 Olofsen E, Daban A. The dynamic relationship between end-tidal sevoflurane and isoflurane concentrations and bispectral index and spectral edge frequency of the electroencephalogram. Anesthesiology 1999; 90: 1345-53

20 Detsch O, Schneider G, Kochs E, Hapfelmeier G, Werner $C$. Increasing isoflurane concentration may cause paradoxical increases in the EEG bispectral index in surgical patients. Br J Anaesth 2000; 84: 33-7.

21 Eger II EI. Isoflurane: a review. Anesthesiology 1981; 55: 559-76.

22 Kanaya N, Nakayama M, Fujita S, Namiki A. Haemodynamic and EEG changes during rapidsequence induction of anaesthesia. Can J Anaesth 1994; 41: 699-702.

23 Nakayama M, Kanaya N, Tsuchida H, Namiki A. The effect of propofol and thiamylal on hypertensive responses to a rapid increase in isoflurane concentration. J Clin Anesth 1999; 11: 8-10.

24 Flaishon R, Windsor A, Sigl J, Sebel PS. Recovery of consciousness after thiopental or propofol. Bispectra index and the isolated forearm technique. Anesthesiology 1997; 86: 613-9. 